\title{
Meta-eQTL: a tool set for flexible eQTL meta-analysis
}

\author{
Antonio Fabio Di Narzo ${ }^{1,2}$, Haoxiang Cheng $^{3}$, Jianwei $\mathrm{Lu}^{4,5^{*}}$ and Ke Hao ${ }^{1,2,5^{*}}$
}

\begin{abstract}
Background: Increasing number of eQTL (Expression Quantitative Trait Loci) datasets facilitate genetics and systems biology research. Meta-analysis tools are in need to jointly analyze datasets of same or similar issue types to improve statistical power especially in trans-eQTL mapping. Meta-analysis framework is also necessary for ChrX eQTL discovery.

Results: We developed a novel tool, meta-eqtl, for fast eQTL meta-analysis of arbitrary sample size and arbitrary number of datasets. Further, this tool accommodates versatile modeling, eg. non-parametric model and mixed effect models. In addition, meta-eqt/ readily handles calculation of chrX eQTLs.

Conclusions: We demonstrated and validated meta-eqtl as fast and comprehensive tool to meta-analyze multiple datasets and ChrX eQTL discovery. Meta-eqtl is a set of command line utilities written in R, with some computationally intensive parts written in C. The software runs on Linux platforms and is designed to intelligently adapt to high performance computing (HPC) cluster. We applied the novel tool to liver and adipose tissue data, and revealed eSNPs underlying diabetes GWAS loci.
\end{abstract}

\section{Background}

Expression quantitative trait loci (eQTLs) are genomic loci that regulate expression levels of mRNAs, and eQTLs play important roles in genetics and systems biology studies. To date, multiple eQTL datasets (where both transcriptome and DNA genotype are profiled on the same individuals) exist for a given tissue type, e.g. liver and lung $[1,2]$. It is necessary to jointly analyze these sets to further improve statistical power especially for trans-eQTL discovery. Even for the same tissue type, the eQTL datasets (transcriptome and genotype data) could be heterogeneous due to platform and lab differences, and meta-analysis (but not pooled analysis) would be the method of choice. Meta-analysis is also desirable the analysis of chromosome X eQTLs in dataset consisting of both males and females. The interpretation of genotype effects on gene expression varies between genders. For example, an allele count of 1 in a female indicates a heterozygote genotype (one reference and one alternative allele), while a count of 1 in a male means

\footnotetext{
*Correspondence: jwlu33@hotmail.com; ke.hao@mssm.edu

${ }^{4} \mathrm{~S} c h o o l$ of Software Engineering and The Advanced Institute of Translational Medicine, Tongji University, Shanghai, China

'Department of Genetics and Genomic Sciences, Icahn School of Medicine at Mount Sinai, New York, NY, USA

Full list of author information is available at the end of the article
}

only alternative allele exists and may cause more profound effects. The variance of the genetic effect may also differ between genders. In such scenario, directly pooling males and females in chromosome X eQTL discovery is invalid, while meta-eQTL tackles this issue elegantly by deriving eQTLs per gender and then combining the test statistics.

The typical strategy of meta-analysis has two steps: (1) calculate and record raw test statistics (e.g. $\beta$ and pvalue) of every transcript-SNP pair per individual dataset, and (2) combine the statistics using meta-analysis approach. However, this strategy is not practical in eQTL setting, where each dataset requires evaluation of $>10^{11}$ tests. Storing the raw statistics of every test is prohibitive due to massive disk and $\mathrm{I} / \mathrm{O}$ demand. The common practice is only recording the top hits (e.g. pvalue $<1 \mathrm{e}-4)$ per dataset and meta-analysis. This strategy will miss the eQTLs that have consistent small-to-moderate effect in multiple datasets [3]. Herein, we propose the solution of parallel and synchronized eQTL computation of multiple datasets, and conducting meta-analysis on the fly. By these means, the above steps (1) and (2) are performed in memory, and only the meta-analysis results which pass a user-defined significance level are outputted to disk. Moreover, $\boldsymbol{m e t a}$-eqt offers versatile features: 
implementation of peak finding algorithm, various statistical models (eg. non-parametric and mixed effect model), consistent handling of missing data, easy deployment on high performance computing (HPC) clusters, etc.

\section{Implementation}

Meta-eqtl is a set of command line utilities written in R, with some computationally intensive parts written in C. Optimized linear algebra code (which is included in the $\mathrm{R}$ package) is used to fit linear models in absence of missing values. When missing values are present, in either the gene expression or SNP data, $\mathrm{C}$ code is called to compute the pairwise minimal sufficient statistics. The data format is based on plain text, tab-delimited files, which make the data easy to inspect and manipulate with standard UNIX utilities. Within meta-eqtl, several modules are dedicated to specific functionality, and can be called individually by user.

Linear regressions meta-analysis is implemented in the $\mathrm{R}$ script lm-meta. The computation occurs in multiple threads, where the number of threads corresponds to the number of datasets in the meta-analysis. The multiple threads proceed concordantly, therefore, the same set of gene expression-SNP tests are evaluated in each individual dataset at the same moment. When statistics are obtained from multiple threads, the meta-analytical test statistic is computed as:

$$
\mathrm{Z}_{\text {meta }}=\left(\sum \mathrm{w}_{\mathrm{k}} \mathrm{Z}_{\mathrm{k}}\right) /\left(\Sigma \mathrm{w}_{\mathrm{k}}\right)^{2 / 2}
$$

where the weights are assigned either based on sample size or the standard error of $\beta$ in each dataset. The software output comprehensive statistics of the fitted model, including effective sample size, regression coefficients, standard error of regression coefficients, transcriptome variance explained (ie, $\left.\mathrm{r}^{2}\right), \mathrm{T}$ statistics $(\boldsymbol{T})$ and pvalues $(\boldsymbol{p})$. The $\boldsymbol{T}$ and $\boldsymbol{p}$ were presented for both meta-analysis and each individual cohort. A separate utility, $l \boldsymbol{m}-\boldsymbol{f} d \boldsymbol{r}$, compares the output from observed and permuted data and quantify FDR. In brief, the meta-analysis results enter the downstream peak finding and empirical FDR calibration by permuting the sample IDs in the gene expression files. To our experience, this empirically estimated FDR is more robust than Benjamini-Hochberg procedure, such as used in MatrixEQTL [4], which is heavily biased when gene expression follows a non-Gaussian distribution. The tool set also contains the eqtl-sex-peaks utility, specially designed for meta-analysis of regression results by gender. kruskal is provided as a non-parametric Kruskal-Wallis test for eQTL detection. Since eQTL computation involves big data sets, gene expression and SNP data are accessed sequentially and concordantly by each thread, and results are reported on the fly, as they are computed. This allows for the analysis of files of arbitrary sample size and arbitrary number of datasets with constant memory usage. Also, this framework enables a natural deployment on HPC and Hadoop clusters as it can trivially distribute the analysis into multiple computing nodes.

\section{Results and discussions}

To our knowledge, meta-eqtl is the first software to perform meta-analysis on arbitrary number of eQTL datasets. We thus compared our results with those obtained with METAL $[5,6]$, a tool which performs meta-analysis on pre-stored test statistics. On a data of four individual sets (sample size of 1000, 1000, 500 and 500, respectively), we tested 10,000 SNPs, and the two software gave the identical results to the available numerical precision. We also benchmarked the performance on a large data of three cohorts $(\mathrm{N}=450,400$ and 350) with 44,000 transcripts profiled and 1000 genome imputed genotype ( $>$ million SNPs). Meta-eqtl distributed the computation on a cluster of 800 computing cores (each core allocated $824 \mathrm{Mb}$ to $1013 \mathrm{Mb}$ of memory), and was able to complete within three days. The top eQTLs (10\% FDR) statistics were identical to those computed by the $R$ package "meta". On a single cohort, we further conducted head-to-head comparison to the MatrixEQTL software [4], which to our knowledge is the fastest software available to date for large scale eQTL analysis, and found that meta-eqtl was about 2-3 times slower, reflecting the expense of missing data handling and the more flexible pipeline. Nevertheless, meta-eqtl is still one of the fastest eQTL tools available.

We also leverage another large-scale published eQTL study data [7], where custom $44 \mathrm{~K}$ RNA microarray were run on 651 liver, 848 adipose fat and 701 subcutaneous fat samples of 1,008 patients. 950 samples from the same

\begin{tabular}{|c|c|c|c|c|}
\hline Tissue & \#eQTL & $\begin{array}{l}\text { \# peak eSNP } \\
\text { in RegDB }\end{array}$ & RegDB enrichment $^{\dagger}$ & \# GWAS loci \\
\hline \multicolumn{5}{|l|}{ Liver } \\
\hline cis & 131 & 80 & $2.36(2.01-2.75)$ & 34 \\
\hline trans & 52 & 9 & $1.98(1.17-3.16)$ & 2 \\
\hline \multicolumn{5}{|c|}{ Omental } \\
\hline cis & 207 & 115 & $2.92(2.56-3.32)$ & 54 \\
\hline trans & 28 & 10 & $1.04(0.64-1.60)$ & 0 \\
\hline \multicolumn{5}{|l|}{ SubQ } \\
\hline cis & 163 & 83 & $3.23(2.81-3.72)$ & 30 \\
\hline trans & 18 & 8 & $1.39(0.80-2.10)$ & 1 \\
\hline
\end{tabular}

*10\% FDR eQTLs on chromosome X. Omental: omental fat tissue, SubQ: Subcutaneous fat tissue; ${ }^{\dagger}$ Enrichment of ChrX eSNP in RegulomeDB database [10], odds ratio (95\% confidence interval) are presented; ${ }^{\mp}$ Number of ChrX eQTLs underlying GWAS loci. 
patients were successfully genotyped on the Illumina 650 Y BeadChip array, and further imputed on the 1000 Genome reference for 14 million SNPs using the MACH [8] pipeline. Applying meta-eQTL, we derived ChrX eQTL for each tissue (Table 1). The meta-analysis of males and females provides increased power in detecting genetic regulation of gene expression, while still correctly keeping separate the analysis of the two sets. In
Figure 1, we illustrate e.g. how the $\mathrm{X}$ chromosome gene DUSP9 shows some evidence of cis-regulation in the liver of both females (top panel) and males (middle panel), with the meta-analytical results pointing to a sharper and more conclusive signal (bottom panel). Further, we employed the ChrX eQTLs to inform type 1 and type 2 diabetes (T1D and T2D) GWAS SNPs (where liver and adipose are disease relevant issues)

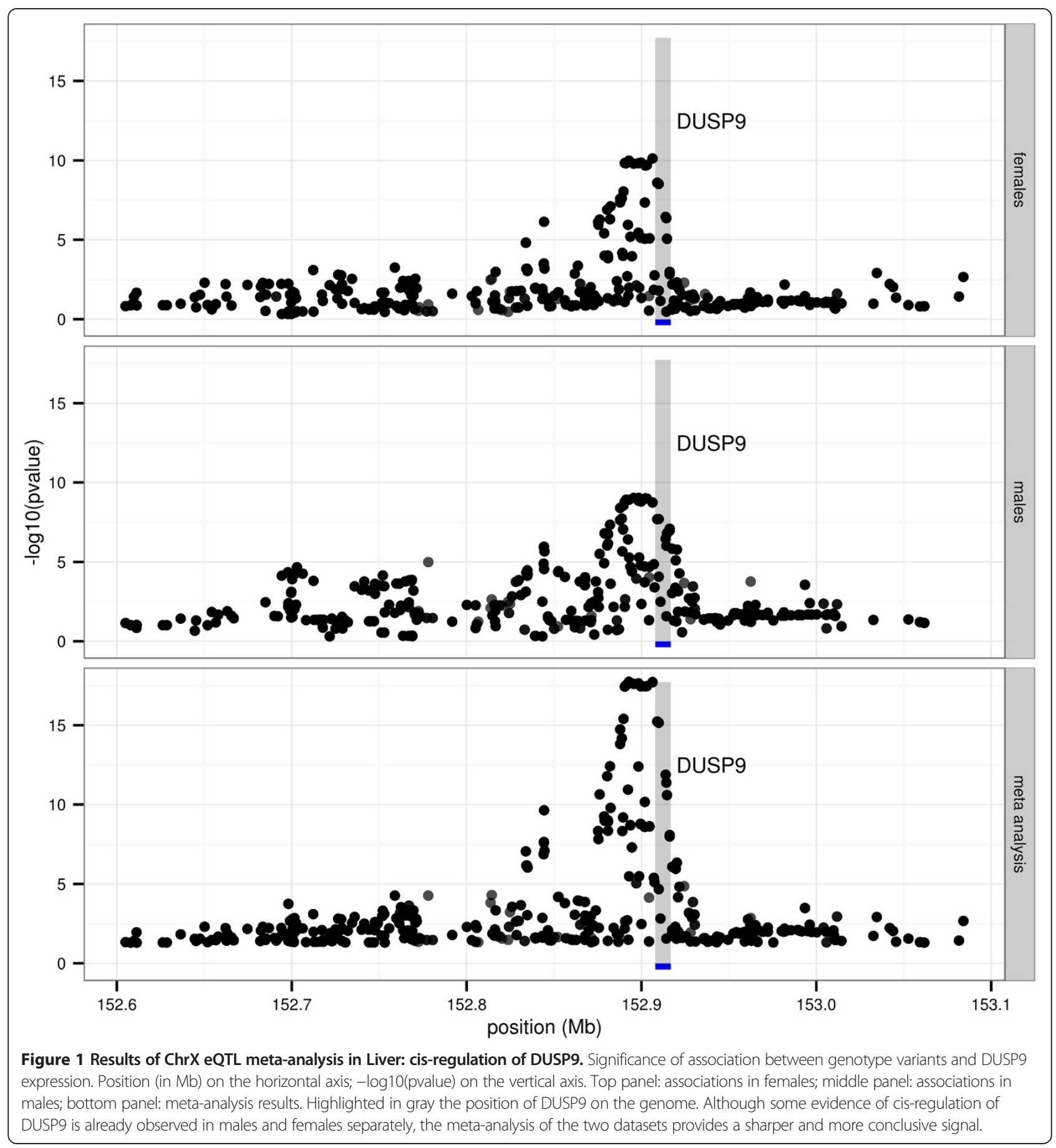


Table 2 ChrX diabetes GWAS hits with significant eQTLs in liver and adipose tissues

\begin{tabular}{lllllll}
\hline rsID & Position & Reported gene & Mapped gene & Disease & Risk Allele & GWAS pvalue \\
\hline rs2664170 & 153945602 & Intergenic & GAB3 & Type 1 diabetes & G & 8e-9 \\
rs5945326 & 152899922 & DUSP9 & KRT18P48 - DUSP9 & Type 2 diabetes & A & 7e-16 \\
rs12010175 & 152862638 & FAM58A & FAM58A & Type 2 diabetes & G & 2e-9 \\
\hline
\end{tabular}

documented in the NHGRI catalog [9]. Three chrX SNPs associated with T1D or T2D were also eQTLs in at least one of these three tissues (Table 2). Genes close to these SNPs were proposed as underlying the disease etiology in the original GWAS reports, herein, we identify additional plausible candidates (Table 3). For examples, rs2664170 is associated with T1D and has profound influence on gene expression levels of IKBKG in all tissues. IKBKG (inhibitor of nuclear factor kappa-B kinase subunit gamma) is the regulatory subunit of the inhibitor of ІкB kinase (IKK) complex, which activates NF- $\mathrm{KB}$ resulting in activation of genes involved in inflammation, immunity, cell survival, and other pathways. Given the inflammatory basis of T1D, IKBKG is a highly relevant genetic risk factor. The direction of eQTL is consistent among the three tissues; that is the disease risk allele (rs2664170-G) is associated with lower level of IKBKG, leading to higher ІкB kinase activity and elevated inflammation and in turn increase T1D risks.

\section{Conclusions}

In summary, we describe a novel package, meta-eqtl. To our knowledge, it is the only tool to allow fast metaanalysis of eQTLs for today's large genotype and gene expression data with reasonable memory requirement and fast speed. It can also be used as a flexible and fast tool for eQTL discover on a single dataset, where it features flexible model specification (e.g. non-parametric and mixed effect models), missing data handling and implements significance peaks extraction. Meta-eqtl features computation speed comparable to the fastest alternative available to date, and is further well suited to distribute parallel jobs onto a HPC system. Another major advantage is the ability to handle chrX eQTLs. In recent year, increasing number of eQTL studies and dataset become available $[3,7,11]$, joint analyses of same/similar tissue sets are of great interest. Meta-eqtl enables meta-analysis of arbitrary number of eQTL dataset and will greatly facilitate this research field.

Table 3 eQTL statistics on ChrX diabetes GWAS hits

\begin{tabular}{|c|c|c|c|c|c|c|c|c|c|}
\hline eQTL Gene & eSNP rsID* & eQTL Eff Allele & eQTL pvalue & $\beta$ & T meta & T male & T female & eQTL Type & Tissue \\
\hline F8 & rs2664170 & A & $3.8 \mathrm{E}-07$ & -3.60 & -5.08 & -2.93 & -4.26 & cis & Liver \\
\hline AK095886 & rs2664170 & A & $9.6 \mathrm{E}-04$ & -2.36 & -3.30 & -0.93 & -3.43 & cis & Liver \\
\hline CTAG2 & rs2664170 & A & $1.2 \mathrm{E}-03$ & 2.35 & 3.25 & 0.69 & 3.46 & cis & Liver \\
\hline IKBKG & rs2664170 & A & $7.4 \mathrm{E}-03$ & 1.92 & 2.68 & 1.07 & 2.53 & cis & Liver \\
\hline CTAG1B & rs2664170 & A & 8.7E-03 & 1.88 & 2.62 & 1.35 & 2.27 & cis & Liver \\
\hline AK095886 & rs2664170 & A & 7.0E-07 & -3.56 & -4.96 & -2.56 & -4.34 & cis & Omental \\
\hline IKBKG & rs2664170 & A & $5.1 \mathrm{E}-05$ & 2.90 & 4.05 & 2.48 & 3.26 & cis & Omental \\
\hline F8A1 & rs2664170 & A & $2.4 \mathrm{E}-04$ & -2.61 & -3.67 & -1.53 & -3.52 & cis & Omental \\
\hline SLC10A3 & rs2664170 & A & 2.7E-04 & 2.59 & 3.64 & 2.99 & 2.30 & cis & Omental \\
\hline AK095886 & rs2664170 & A & $8.1 \mathrm{E}-07$ & -3.52 & -4.93 & -2.88 & -4.10 & cis & SubQ \\
\hline IKBKG & rs2664170 & A & $5.5 \mathrm{E}-04$ & 2.45 & 3.46 & 3.35 & 1.77 & cis & SubQ \\
\hline Contig21200_RC & rs2664170 & A & $4.3 \mathrm{E}-08$ & -3.91 & -5.48 & -2.69 & -4.96 & trans & SubQ \\
\hline XM_210086 & rs5945326 & A & $7.0 \mathrm{E}-03$ & 1.94 & 2.70 & 0.61 & 2.86 & cis & Liver \\
\hline SLC6A8 & rs12010175 & G & $2.8 \mathrm{E}-04$ & 2.62 & 3.63 & 2.99 & 2.41 & cis & Liver \\
\hline ARHGAP4 & rs12010175 & G & $8.2 \mathrm{E}-03$ & 1.91 & 2.64 & 0.13 & 3.08 & cis & Liver \\
\hline BC030106 & rs12010175 & G & $1.8 \mathrm{E}-03$ & 2.28 & 3.12 & 2.81 & 2.00 & cis & Omental \\
\hline HSS00085101 & rs12010175 & G & $3.2 \mathrm{E}-03$ & -2.11 & -2.94 & -1.15 & -2.82 & cis & Omental \\
\hline PLXNB3 & rs12010175 & G & $3.0 \mathrm{E}-04$ & 2.62 & 3.61 & 1.42 & 3.41 & cis & SubQ \\
\hline BC030106 & rs12010175 & G & $7.6 \mathrm{E}-04$ & 2.38 & 3.37 & 2.07 & 2.71 & cis & SubQ \\
\hline
\end{tabular}

*SNPs rs2664170, rs5945326 and rs12010175 were identified as eQTL, and these SNPs are also reported in association with diabetes by large GWA studies (summarized in Table 2). 


\section{Availability and requirements}

The meta-eQTL software package is freely available to all readers under https://haok01.u.hpc.mssm.edu/meta_eQTL/

Project name: meta-eQTL

Project home page: https://haok01.u.hpc.mssm.edu/ meta_eQTL/

Operating system(s): Linux

Programming language: $\mathrm{R}$ (version 3.0 .2 ) and $\mathrm{C}$

License: none

\section{Any restrictions to use by non-academics: none}

\section{Competing interests}

The authors declare that they have no competing interests.

\section{Authors' contributions}

DNA, $C H, L$ and $H K$ are responsible for designing and conducting the study. DNA, $L$ and HK wrote the manuscript. All authors read and approved the final manuscript.

\section{Acknowledgements}

This work is supported by NIH grant R01HL118542, 973 Program (No. 2013CB967101) of the Ministry of Science Technology of China, Shanghai Science Committee Foundation (13PJ1433200) and National Natural Science Foundation of China (Grant No. 21477087).

\section{Author details}

${ }^{1}$ Department of Genetics and Genomic Sciences, Icahn School of Medicine at Mount Sinai, New York, NY, USA. ${ }^{2}$ Icahn Institute of Genomics and Multiscale Biology Icahn School of Medicine at Mount Sinai, New York, NY, USA. ${ }^{3}$ College of Electronics and Information Engineering, CIMS Research Center, Tongji University, Shanghai, China. ${ }^{4}$ School of Software Engineering and The Advanced Institute of Translational Medicine, Tongji University, Shanghai, China. ${ }^{5}$ Department of Respiratory Medicine, Shanghai Tenth People's Hospital, Tongji University, Shanghai, China.

Received: 3 July 2014 Accepted: 19 November 2014

Published online: 28 November 2014

\section{References}

1. Schadt EE, Molony C, Chudin E, Hao K, Yang X, Lum P, Kasarskis A, Zhang B, Wang S, Suver C, Zhu J, Millstein J, Sieberts S, Lamb J, GuhaThakurta D, Derry J, Storey JD, Avila-Campillo I, Kruger MJ, Johnson JM, Rohl CA, van Nas A, Mehrabian M, Drake TA, Lusis AJ, Smith RC, Guengerich FP, Strom SC, Schuetz E, Rushmore TH, et al: Mapping the genetic architecture of gene expression in human liver. PLOS Biol 2008, 6:e107.

2. Hao K, Bosse $Y$, Nickle DC, Paré PD, Postma DS, Laviolette M, Sandford A Hackett TL, Daley D, Hogg JC, Elliott WM, Couture C, Lamontagne M, Brandsma CA, van den Berge M, Koppelman G, Reicin AS, Nicholson DW Malkov V, Derry JM, Suver C, Tsou JA, Kulkarni A, Zhang C, Vessey R, Opiteck GJ, Curtis SP, Timens W, Sin DD: Lung eQTLs to help reveal the molecular underpinnings of asthma. PLoS Genet 2012, 8:e1003029.

3. Liang L, Morar N, Dixon AL, Lathrop GM, Abecasis GR, Moffatt MF, Cookson WOC: A cross-platform analysis of 14,177 expression quantitative trait loci derived from lymphoblastoid cell lines. Genome Res 2013, 23:716-726.

4. Shabalin AA: Matrix eQTL: ultra fast eQTL analysis via large matrix operations. Bioinformatics 2012, 28:1353-1358.

5. Sanna S, Jackson AU, Nagaraja R, Willer CJ, Chen WM, Bonnycastle LL, Shen H, Timpson N, Lettre G, Usala G, Chines PS, Stringham HM, Scott L, Dei M, Lai S, Albai G, Crisponi L, Naitza S, Doheny KF, Pugh EW, Ben-Shlomo Y, Ebrahim S, Lawlor DA, Bergman RN, Watanabe RM, Uda M, Tuomilehto J, Coresh J, Hirschhorn JN, Shuldiner AR, et al: Common variants in the GDF5-UQCC region are associated with variation in human height. Nat Genet 2008, 40:198-203.

6. Willer CJ, Sanna S, Jackson AU, Scuteri A, Bonnycastle LL, Clarke R, Heath SC, Timpson NJ, Najjar SS, Stringham HM, Strait J, Duren WL, Maschio A, Busonero F, Mulas A, Albai G, Swift AJ, Morken MA, Narisu N, Bennett D, Parish S, Shen H, Galan P, Meneton P, Hercberg S, Zelenika D, Chen WM Li Y, Scott $L$, Scheet PA: Newly identified loci that influence lipid concentrations and risk of coronary artery disease. Nat Genet 2008, 40:161-169.

7. Greenawalt DM, Dobrin R, Chudin E, Hatoum IJ, Suver C, Beaulaurier J, Zhang B, Castro V, Zhu J, Sieberts SK, Wang S, Molony C, Heymsfield SB, Kemp DM, Reitman ML, Lum PY, Schadt EE, Kaplan LM: A survey of the genetics of stomach, liver, and adipose gene expression from a morbidly obese cohort. Genome Res 2011, 21:1008-1016.

8. Howie B, Fuchsberger C, Stephens M, Marchini J, Abecasis GR: Fast and accurate genotype imputation in genome-wide association studies through pre-phasing. Nat Genet 2012, 44:955-959.

9. Hindorff LA, Sethupathy P, Junkins HA, Ramos EM, Mehta JP, Collins FS, Manolio TA: Potential etiologic and functional implications of genomewide association loci for human diseases and traits. Proc Natl Acad Sci U S A 2009, 106:9362-9367.

10. Boyle AP, Hong EL, Hariharan M, Cheng Y, Schaub MA, Kasowski M, Karczewski K, Park J, Hitz BC, Weng S, Cherry JM, Snyder M: Annotation of functional variation in personal genomes using RegulomeDB. Genome Res 2012, 22:1790-1797.

11. Emilsson V, Thorleifsson G, Zhang B, Leonardson AS, Zink F, Zhu J, Carlson S, Helgason A, Walters GB, Gunnarsdottir S, Mouy M, Steinthorsdottir V, Eiriksdottir GH, Bjornsdottir G, Reynisdottir I, Gudbjartsson D, Helgadottir A, Jonasdottir A, Jonasdottir A, Styrkarsdottir U, Gretarsdottir S, Magnusson KP, Stefansson H, Fossdal R, Kristjansson K, Gislason HG, Stefansson T, Leifsson BG, Thorsteinsdottir U, Lamb JR, et al: Genetics of gene expression and its effect on disease. Nature 2008, 452:423-428.

doi:10.1186/s12859-014-0392-0

Cite this article as: Di Narzo et al:: Meta-eQTL: a tool set for flexible eQTL meta-analysis. BMC Bioinformatics 2014 15:392.

\section{Submit your next manuscript to BioMed Central and take full advantage of:}

- Convenient online submission

- Thorough peer review

- No space constraints or color figure charges

- Immediate publication on acceptance

- Inclusion in PubMed, CAS, Scopus and Google Scholar

- Research which is freely available for redistribution 